\title{
Selection of parameters in iris recognition system
}

\author{
Tomasz Marciniak • Adam Dąbrowski • Agata Chmielewska • \\ Agnieszka Anna Krzykowska
}

(C) The Author(s) 2012. This article is published with open access at Springerlink.com

\begin{abstract}
This paper presents the detailed analysis of implementation issues occurred during preparation of the novel iris recognition system. First, we shortly describe the currently available acquisition systems and databases of iris images, which were used for our tests. Next, we concentrate on the feature extraction and coding with the execution time analysis. Results of the average execution time of loading the image, segmentation, normalization, and feature encoding, are presented. Finally, DET plots illustrate the recognition accuracy for IrisBath database.
\end{abstract}

Keywords Iris recognition $\cdot$ Biometric $\cdot$ CASIA $\cdot$ IrisBath

\section{Introduction}

Identification techniques based on the iris analysis gained popularity and scientific interest since John Daugman introduced in 1993 the first algorithm for the identification of persons based on the iris of the eye [5]. Then many other researchers presented new solutions in this area [3, 13, 14, 16, 21, 23, 25-27].

This paper was prepared within the INDECT project.

T. Marciniak · A. Dąbrowski · A. Chmielewska · A. A. Krzykowska (凶)

Division of Signal Processing and Electronic Systems, Chair of Control

and Systems Engineering, Poznań University of Technology, ul.Piotrowo 3a,

60-965 Poznań, Poland

e-mail: agnieszka.krzykowska@put.poznan.pl

T. Marciniak

e-mail: tomasz.marciniak@put.poznan.pl

A. Dąbrowski

e-mail: adam.dabrowski@put.poznan.pl

A. Chmielewska

e-mail: agata.chmielewska@put.poznan.pl 
The iris is an element, which arises already in an early phase of the human life and remains unchanged for a long part of the life. Construction of the iris is independent of the genetic relationships and each person in the world, even the twins possess different irises. However, there are also many problems to be faced when encoding the iris such as a change of the opening angle in the pupil depending on lighting conditions, covering a portion of its regions by the eyelids and eyelashes, or the rotation of the iris due to the inclination of the head or eye movement.

\section{Acquisition of iris images and iris databases}

Generally acquisition of the iris should be implemented in accordance with the standards. The application interface has to be built using ANSI INCITS 358-2002 (known as BioAPI ${ }^{\mathrm{TM}}$ v1.1) recommendations. Additionally, the iris image should comply with ISO/IEC 19794-6 norm [1].

One of the first systems for the iris acquisition were developed using concepts proposed by Daugman [5] and Wildes [26]. Daugman's system performs image of the iris with a diameter of typically between 100 and 200 pixels, taking pictures from a distance of $15-46 \mathrm{~cm}$, using a $330 \mathrm{~mm}$ lens. In the case of the Wildes proposal the iris image has a diameter of about 256 pixels and the photo is taken with a distance of $20 \mathrm{~cm}$ using a $80 \mathrm{~mm}$ lens.

Currently, several iris acquisition devices as well as whole iris recognition systems can be found on the commercial market. Examples of the iris devices are shown in Table 1.

There are systems, which enable detection of the iris of persons who are in motion, too. In 2009, the company Sarnoff [9] presented the first device in the series IrisOn-the-Move, which realizes this assumption. The IOM Passport Portal System allows the detection and identification of thirty people per minute. The system can effectively be used to secure the objects with a large flow of people, such as embassies, airports, or factories. Figure 1 shows the diagram of the IOM system. The system uses a card reader, which is a preliminary step in persons identification. The person is detected by the system of cameras, then the iris is detected and the iris code is determined, which is compared with the pattern. An advantage of this system is its ability to identify people who wear glasses or contact lenses. The system allows to identify people from the distance of three meters.

Experimental studies were carried out using the databases containing photos of irises prepared by scientific institutions dealing with this issue. Two publicly available databases were used during our experiments, as shown in Section 4. The first database was CASIA [2], coming from the Chinese Academy of Sciences, Institute of Automation, while the second IrisBath [24] was developed at the University of Bath. We have also obtained an access to UBIRIS v.2.0 database [22] and the database prepared by Michael Dobeš and Libor Machala [7].

CASIA database is presented in three versions. All present photographs were taken in the near infrared. We used the first and the third version of this database in our experimental research. Version 1.0 contains 756 iris images with dimensions $320 \times 280$ pixels carried out on 108 different eyes. The pictures in CASIA database were taken using the specialized camera and saved in BMP format. For each eye 7 photos were made, 3 in the first session and 4 in the second. Pupil area was 


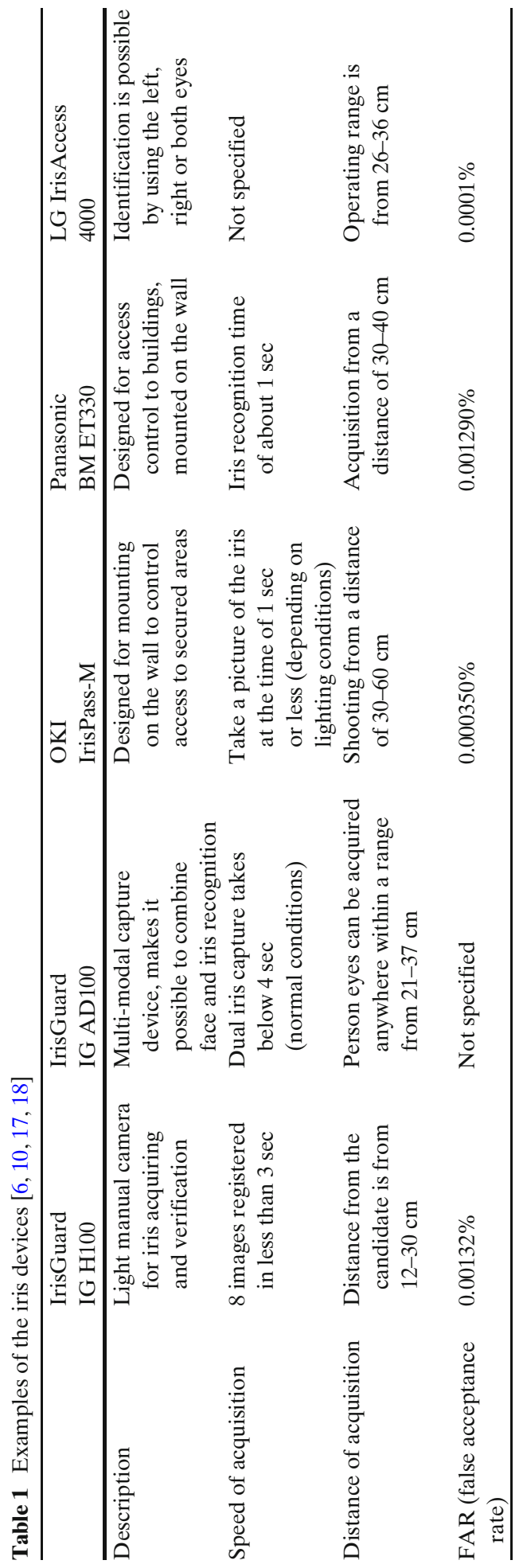


Fig. 1 Diagram of the system: Iris-On-the-Move

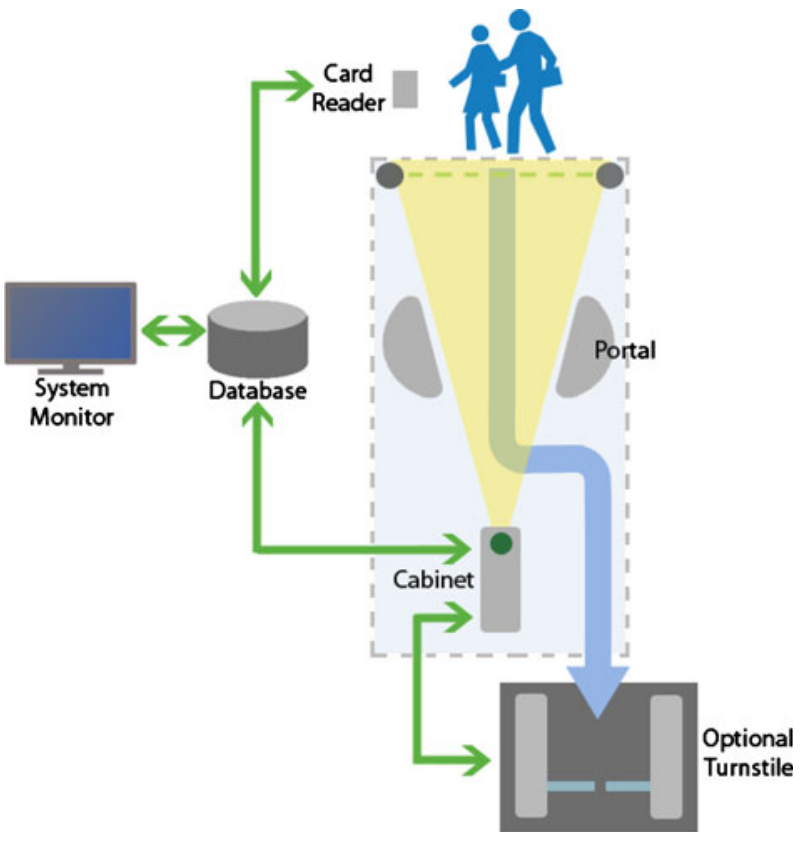

uniformly covered with a dark color, thus eliminating the reflections occurring during the acquisition process.

The third version of the CASIA database contains more than 22,000 images from more than 700 different objects. It consists of three sets of data in JPG 8-bit format. Section of CASI-IrisV3-Lamp contains photographs taken at the turned-on and off lamp close to the light source to vary the lighting conditions, while the CASIA-IrisV3 Twins includes images of irises of hundred pairs of twins.

Lately a new version of CASIA database has been created the CASIA-IrisV4. It is an extension of the CASIA-IrisV3 and contains six subsets. Three subsets from CASIA-IrisV3 are: CASIA-Iris-Interval, CASIA-Iris-Lamp, and CASIA-Iris-Twins. Three new subsets are: CASIA-Iris-Distance, CASIA-Iris-Thousand, and CASIAIris-Syn.

CASIA-Iris-Distance contains iris images captured using self-developed longrange multi-modal biometric image acquisition and recognition system. The advanced biometric sensor can recognize users from $3 \mathrm{~m}$ away. CASIA-Iris-Thousand contains 20,000 iris images from 1,000 subjects. CASIA-Iris-Syn contains 10,000 synthesized iris images of 1,000 classes. The iris textures of these images are synthesized automatically from a subset of CASIA-IrisV1.

CASIA-IrisV4 contains a total of 54,607 iris images from more than 1800 genuine subjects and 1,000 virtual subjects. All iris images are 8-bit gray-level JPEG files, collected under the near infrared illumination.

The IrisBath database is created by the Signal and Image Processing Group (SIPG) at the University of Bath in the UK [24]. The project aimed to bring together 20 high resolution images from 800 objects. Most of the photos show the irises of students from over one hundred countries, who form a representative group. The photos were performed with the resolution of 1,280 $\times 960$ pixels in 8 -bit BMP, using 
a system with camera LightWise ISG. There are thousands of free of charge images that have been compressed into the JPEG2000 format with the resolution of 0.5 bit per pixel.

\section{Extraction and coding of iris features}

We can identify three successive phases in the process of creating the iris code [15]. They are determined respectively as: segmentation, normalization, and features encoding as shown in Fig. 2.

\subsection{Segmentation process}

Separation of the iris from the whole eye area is realized during the segmentation phase. At this stage it is crucial to determine the position of the upper and lower eyelids, as well as the exclusion of areas covered by the lashes. In addition, an attention should be paid to the elimination of regions caused by the light reflections from the cornea of the eye.

The first technique of iris location was proposed by the precursor in the field of the iris recognition i.e. by Daugman [5]. This technique uses the so-called integrodifferential operator, which acts directly on the image of the iris, seeking the maximum normalized standard circle along the path, a partial derivative of the blurred image relating to the increase of the circle radius. The current operator behaves like a circular edge detector in the picture, acting in the three-dimensional parameter space $(x, y, r)$, i.e. the center of the coordinates and the radius of the circle are looked for, which determine the edge of the iris. The algorithm detects first the outer edge of the iris, and then, limited to the area of the detected iris, it is looking for its inside edge. Using the same operator, but by changing the contour of the arc path, we can also look for the edges of the eyelids, which may in part overlap the photographed iris.

Fig. 2 Stages of creation of iris codes

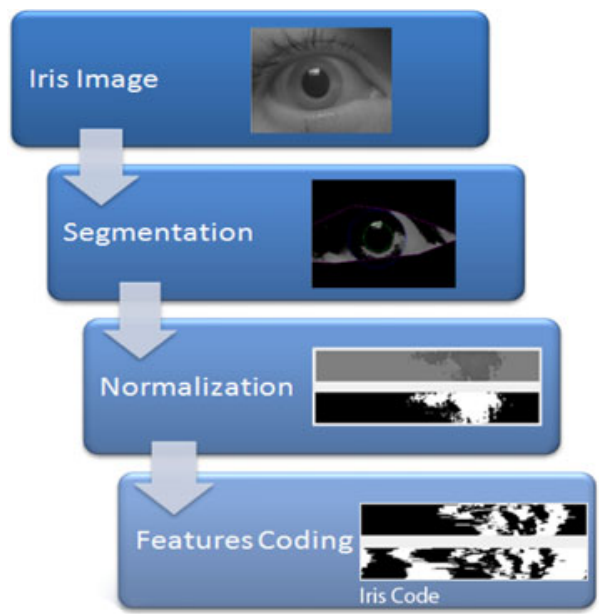

Springer 
Another technique was proposed by Wildes [26]. In this case also the best fit circle is looked for but the difference (comparing to the Daugman method) consists in a way of searching the parameter space. Iris localization process takes in this case place in two stages. First, the image edge map is created then each detected edge point gives a vote to the respective values in the parameter space looking for the pattern. The edge map is created based on the gradient method. It relies on the assignment of a scalar bitmap vector field, defining the direction and strength increase in the pixel brightness. Then, the highest points of the gradient, which determine the edges, are left with an appropriately chosen threshold. The voting process is performed at the designated edge map using the Hough transform.

In our experimental program [11] we also used the Hough transform, and to designate the edge map we used the modified Kovesi algorithm [12] based on the Canny edge detector. An illustration of the segmentation process with the execution time analysis is presented in Fig. 3.

\subsection{Normalization}

The main aim of the normalization step is the transformation of the localized iris to a defined format in order to allow comparisons with other iris codes. This operation requires consideration of the specific characteristics of the iris like a variable pupil opening, non coordinated pupil, and the iris center points. A possibility of circulation of the iris by tilting the head or as a result of the eye movement in an orbit, should be noticed.

Having successfully located the image area occupied by the iris, the normalization process has to ensure that the same areas at different iris images are represented in the same scale in the same place of the created code. Only with equal representations the comparing two iris codes can be correctly justified. Daugman suggested a standard transformation from Cartesian coordinates to the ring in this phase. This transformation eliminates the problem of the non-central position of the pupil relatively to the iris as well as the pupil opening variations with different lighting conditions. For further processing, points contained in the vicinity of a 90 and $270^{\circ}$ (i.e., at the top and at the bottom of the iris) can be omitted, This reduces errors caused by the presence of the eyelids and eyelashes in the iris area.

Poursaberi [20] proposed to normalize just only half of the iris (close to the pupil), thus by passing the problem of the eyelids and eyelashes. Pereira [19] showed, in the experiment, in which the iris region was divided into ten rings of equal width, that the potentially better decision can be made with only a part of the rings, namely those numbered as $2,3,4,5,7$, with the ring numbered as the first one, being the closest to the pupil.

During our tests, the Daugman proposal and the model based on its implementation by Libor Masek in Matlab [15] was used in the step normalization stage. At the same time we can select an area of the iris, which is subject to normalization, using both angular distribution and the distribution along the radius. The division consists in determining the angular range of orientation, which is made with the normalization of the iris. This range is defined in two intervals: the first includes angles from -90 to $90^{\circ}$ and the second-the angles from 90 to $-90^{\circ}$ (i.e., angles opposite to clockwise). 


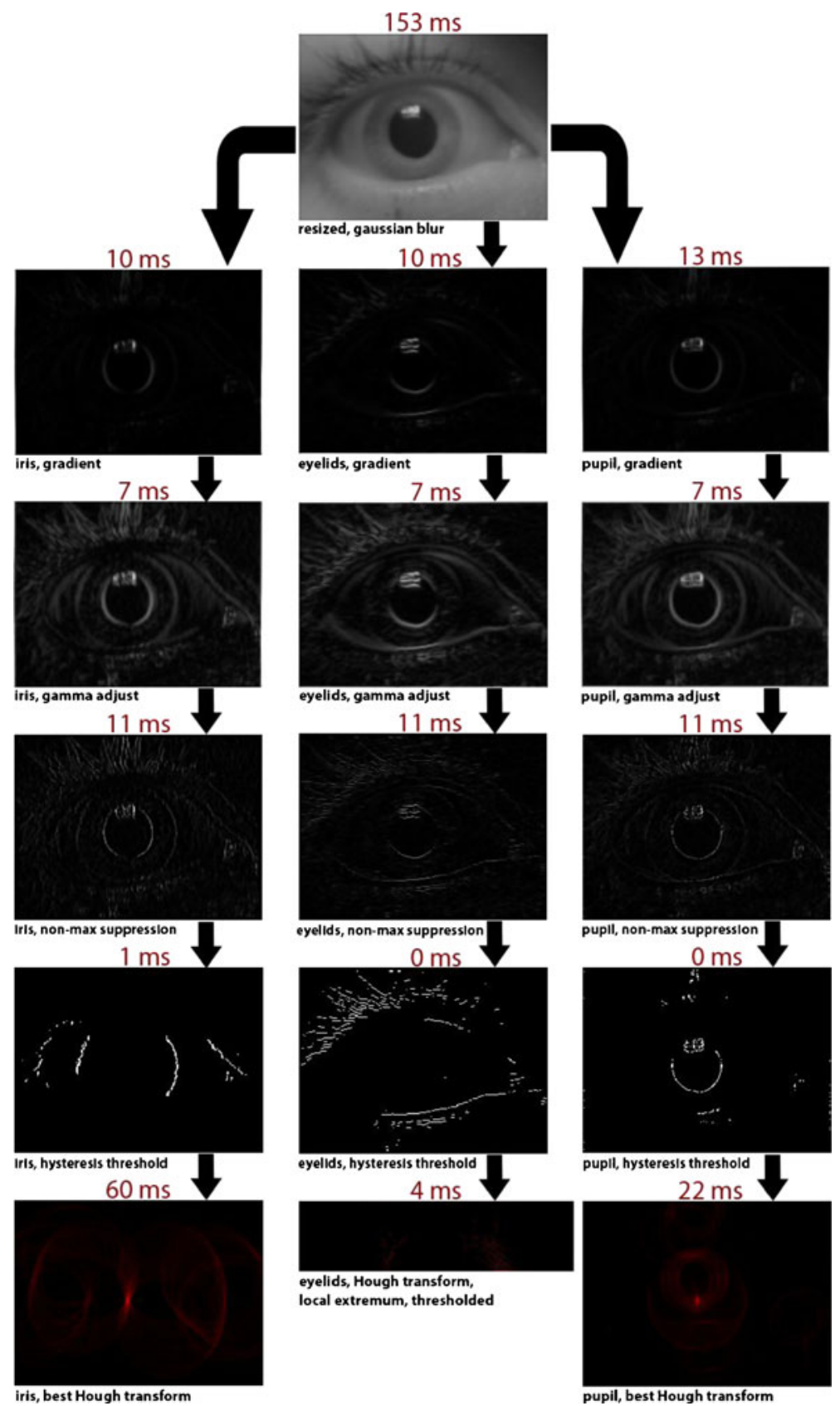

Fig. 3 Example time-consuming analysis of segmentation process

\subsection{Features coding}

The last stage of the feature extraction, which encode the characteristics, aims to extract the normalized iris distinctive features of the individual and to transform them 
into a binary code. In order to extract individual characteristics of the normalized iris, various types of filtering can be applied. Daugman coded each point of the iris with two bits using two-dimensional Gabor filters and quadrature quantization.

Field suggested using a variety logarithmic Gabor filters, the so called Log-Gabor filters [8]. These filters have certain advantages over and above conventional Gabor filters, namely, by definition, they do not possess a DC component, which may occur in the real part of the Gabor filters. Another advantage of the logarithmic variation is that it exposes high frequencies over low-frequencies. This mechanism approaches the nature of these filters to a typical frequency distribution in real images. Due to this feature the logarithmic Gabor filters better expose information contained in the image.

\section{Time analysis}

During our research we used the program IrisCode_TK2007 [11]. A multi-processing was used in order to automatically create iris codes for multiple files. The study involved two databases described in Section 2, namely CASIA and IrisBath.

Test results are presented in Table 2. Section "Information" includes the total number of files and the number of classes of irises. Section "Results" contains the results of the processed images. These are average execution times of individual stages and the total processing time for all files. Figure 4 shows the times of individual

Table 2 Processing times for two bases: IrisBath and CASIA

\begin{tabular}{|c|c|c|c|c|c|c|c|}
\hline \multirow[t]{3}{*}{ Data base name } & \multicolumn{5}{|l|}{ CASIA } & \multirow[t]{3}{*}{ IrisBath } & \multirow{3}{*}{$\begin{array}{l}\text { All } \\
\text { databases }\end{array}$} \\
\hline & \multirow{2}{*}{$\begin{array}{l}\text { Iris image } \\
\text { Database } \\
(\mathrm{v} 1.0)\end{array}$} & \multicolumn{3}{|l|}{ IrisV3 } & \multirow{2}{*}{$\frac{\text { Iris V4 }}{\text { Distance }}$} & & \\
\hline & & Interval & Lamp & Twins & & & \\
\hline \multicolumn{8}{|l|}{ Information } \\
\hline $\begin{array}{l}\text { Number of classes } \\
\text { of irises }\end{array}$ & 217 & 498 & 822 & 400 & 142 & 22 & 2,101 \\
\hline Total number of files & 756 & 2,655 & 16,213 & 3,183 & 2,572 & 432 & 25,811 \\
\hline \multicolumn{8}{|l|}{ Results (times) } \\
\hline $\begin{array}{l}\text { The average time of } \\
\text { image loading [ms] }\end{array}$ & 87 & 86 & 278 & 283 & 3,956 & 1,120 & 968 \\
\hline $\begin{array}{l}\text { The average time of } \\
\text { segmentation }[\mathrm{ms}]\end{array}$ & 103 & 107 & 320 & 306 & 4,404 & 1,249 & 1,081 \\
\hline $\begin{array}{l}\text { The average time of } \\
\text { normalization [ms] }\end{array}$ & 2 & 2 & 2 & 2 & 2 & 2 & 2 \\
\hline $\begin{array}{l}\text { The average time } \\
\text { of features } \\
\text { encoding [ms] }\end{array}$ & 1 & 1 & 1 & 1 & 2 & 1 & 1 \\
\hline $\begin{array}{l}\text { The average total } \\
\text { time coding [ms] }\end{array}$ & 193 & 196 & 601 & 592 & 8,364 & 2,372 & 2,053 \\
\hline $\begin{array}{l}\text { The average time of } \\
\text { writing results } \\
\text { on disc [ms] }\end{array}$ & 6 & 5 & 6 & 6 & 8 & 5 & 6 \\
\hline $\begin{array}{l}\text { Total time database } \\
\text { processing }\end{array}$ & 00:02:30 & 00:08:55 & $02: 43: 57$ & $00: 31: 46$ & 06:10:15 & $00: 17: 10$ & 09:54:28 \\
\hline
\end{tabular}


Fig. 4 Participation of particular stages, expressed in percentage, for analyzed tested databases

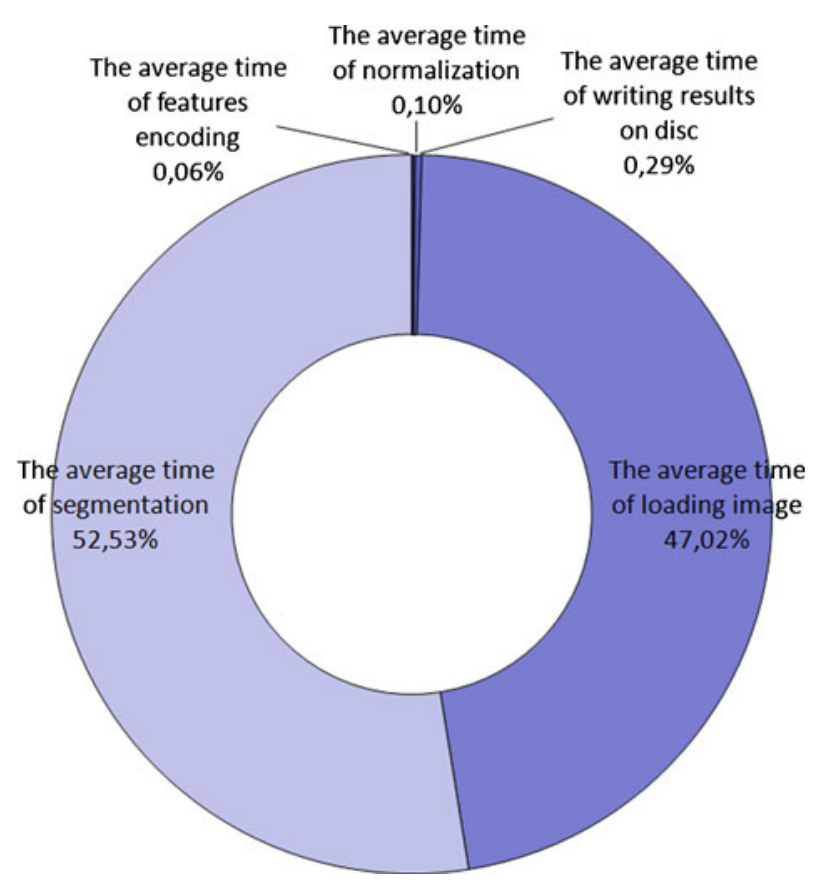

stages, expressed in percentage of the overall time for all tested databases (processed with Intel Core i7 CPU; 2,93 GHz).

Our program contains also an option "Multithreading", which enables multithreaded processing on multiprocessor machines. Figure 5 presents the comparison of the processing times of various stages, when the option "Multithreading" was used or not (processed on Intel Core i7 CPU; $2.93 \mathrm{GHz}$ ) for IrisBath database. The total processing time for one processor was about $17 \mathrm{~min}$. while for two processors was about $9 \mathrm{~min}$.

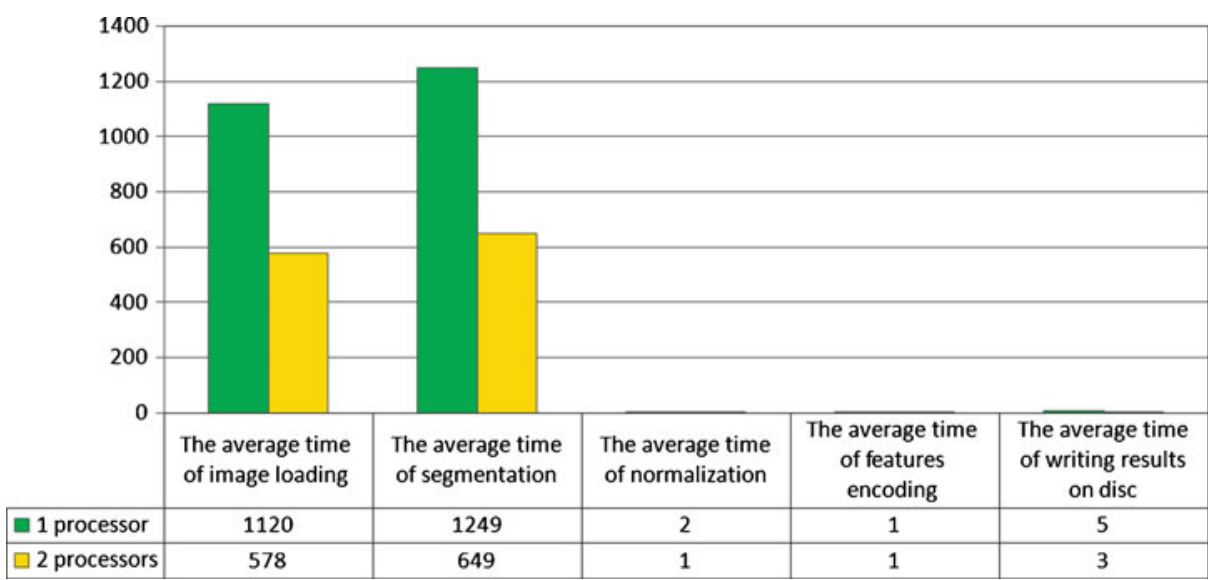

Fig. 5 Participation of individual stages, expressed in percentage, for all tested databases 
Fig. 6 Area of normalization

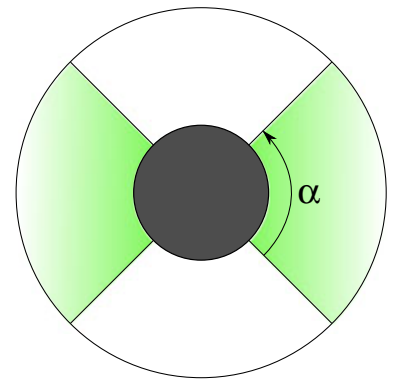

(a) Angular span

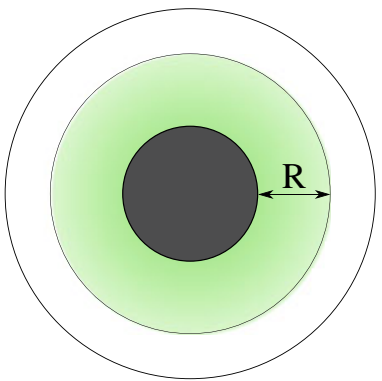

(b) Radius span

\section{Results of identification with IrisBath database}

\subsection{Study of the areas of normalization}

In this experiment we tested dependancy of the iris identification from different parts of the iris.

First, we examined how particular angular span of the iris influences identification of a person. We define angular span as a range of the iris that was used for normalization. Two semicircles were obtained by dividing the circle describing the iris with a vertical line. In each of the semicircles we define angles of $n$ degrees oriented in opposite directions to receive areas of normalization as shown in Fig. 6a.

The second part of the experiment included study of the impact that length of the radius of iris has on person identification. A length of the radius of iris defines a segment of the iris that is used for normalization. Such ring does not have to start on

Fig. 7 DET plots illustrating influence of angular span of iris on person identification

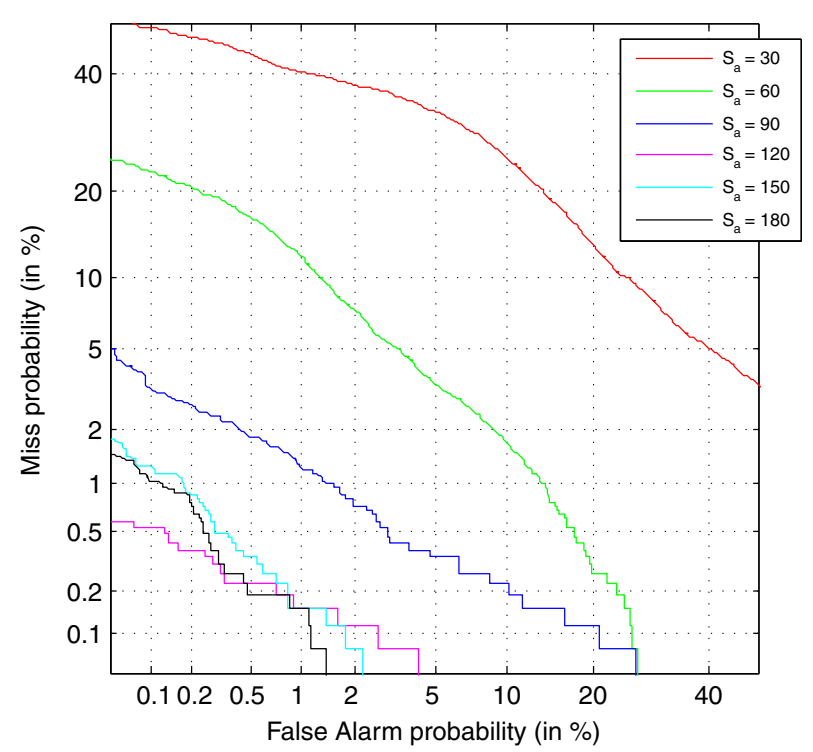


Table 3 EER for particular angular span of iris normalization area

\begin{tabular}{lllllll}
\hline Angular span $S_{a}\left[^{\circ}\right]$ & 30 & 60 & 90 & 120 & 150 & 180 \\
\hline EER (equal error rate) [\%] & 0.1652 & 0.0408 & 0.0118 & 0.0031 & 0.0040 & 0.0031 \\
\hline
\end{tabular}

the edge of pupil and does not have to end on the outer edge of the iris. Figure $6 \mathrm{~b}$ illustrates the idea of this approach.

\subsubsection{Angular span}

For this experiment a symmetric areas of iris were used, with angles ranged from 30 to $180^{\circ}$ (with $30^{\circ}$ interval). Figure 7 shows results of this experiment and Table 3 shows the obtained EER values.

It can be observed that the best results of the iris identification where obtained for an angle in range from 120 to $180^{\circ}$, which is the biggest possible value of this parameter. From Fig. 6 it can also be inferred that the increasing angular spanning from 120 to $180^{\circ}$ does not give much of improvement. Based on this, we can conclude that the upper and the lower parts of the iris (very near to the outer edge) do not include significant information and are in most cases covered by lids and lashes.

\subsubsection{Length of radius}

After defining the original radius $R$ of iris as a part from its inter to outer edge, two different experiments can be taken into consideration. First, we tested how the length of the radius that is increasing from the inter to the outer edge influences identification of a person. In the second step, the rings of the iris from its outer part were studied. Results of those experiments are shown in Figs. 8 and 9, respectively.

Fig. 8 DET plots illustrating influence of length of radius of the iris on person identification (radius increases from inside to outside)

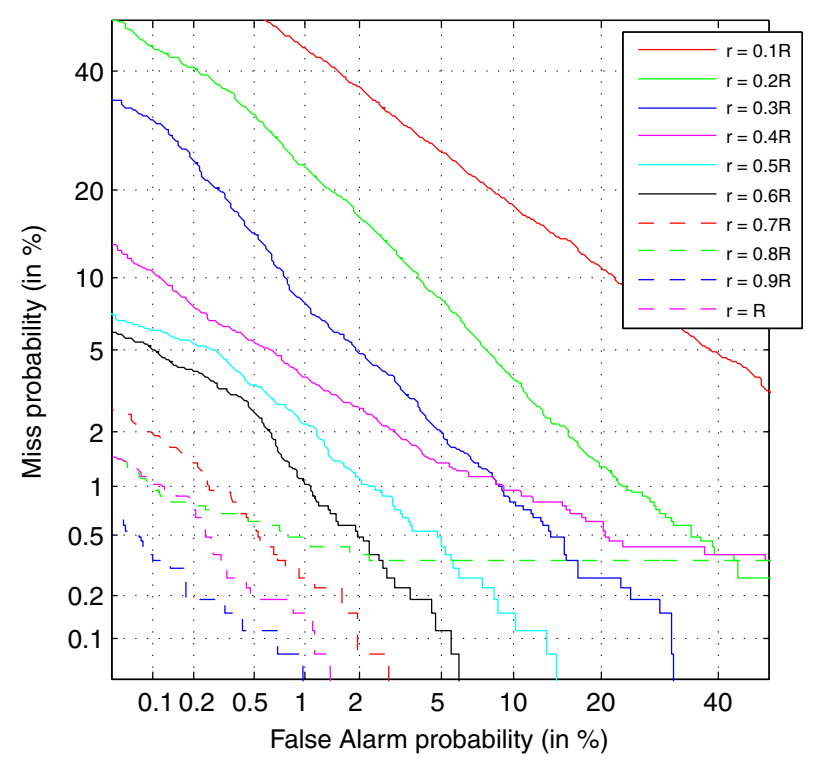


Fig. 9 DET plots illustrating influence of length of radius of the iris on person identification (radius increases from outside to inside)

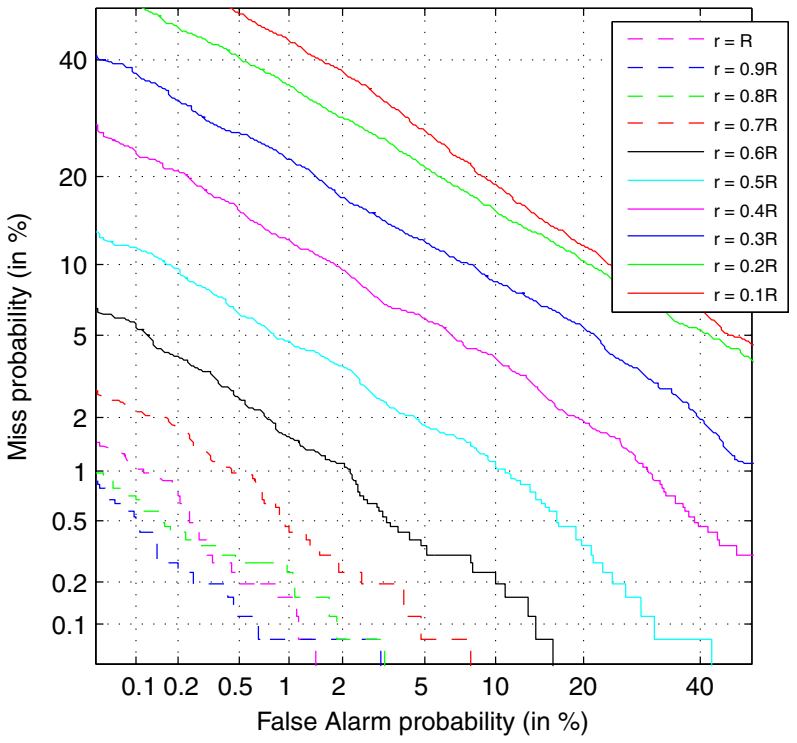

Table 4 contains EERs for these experiments. The tests were performed for the angular span equal to $180^{\circ}$.

From Fig. 8 and Table 4 it can be seen that thicker rings of the iris image used for normalization give better results when increasing the radius from inside to outside, but only up to $r=0.9 R$. If the whole iris image is taken, the result is worse. It can be inferred that the outer parts $(r \in[0.9 R ; R])$ of the iris are covered with lids or lashes and that impedes identification.

Furthermore from Fig. 9 it can be deduced that the same length of radius (for $r \in[0.1 R ; 0.5 R])$ gives better results for inner parts of the iris. This leads to the conclusion that the outer parts of iris do not contain the same amount of distinctive information as the inner parts. Another observation is the fact that far inner part of the iris can have negative impact on the person identification based on Fig. 8. Such phenomenon may be caused by vicinity of pupil.

Table 4 EER for particular radii of the iris used for iris normalization

\begin{tabular}{lll}
\hline $\begin{array}{l}\text { Radius of iris area used } \\
\text { for normalization }\end{array}$ & $\begin{array}{l}\text { EER (equal error rate) [\%] } \\
\text { for increase of radius } \\
\text { from inside to outside }\end{array}$ & $\begin{array}{l}\text { EER (equal error rate) [\%] } \\
\text { for increase of radius } \\
\text { from outside to inside }\end{array}$ \\
\hline$r=0.1 R$ & 0.1419 & 0.1453 \\
$r=0.2 R$ & 0.0639 & 0.1323 \\
$r=0.3 R$ & 0.0329 & 0.0904 \\
$r=0.4 R$ & 0.0236 & 0.0565 \\
$r=0.5 R$ & 0.0142 & 0.0273 \\
$r=0.6 R$ & 0.0103 & 0.0133 \\
$r=0.7 R$ & 0.0053 & 0.0071 \\
$r=0.8 R$ & 0.0057 & 0.0034 \\
$r=0.9 R$ & 0.0019 & 0.0023 \\
$r=R$ & 0.0031 & 0.0031 \\
\hline
\end{tabular}




\section{Conclusions}

The most important issue in the biometric identification process is recognition accuracy. In iris recognition system it depends on acquisition precision and features extraction parameters.

During our study the following results were obtained: FAR $=0.351 \%$ (false acceptance rate) and $\mathrm{FRR}=0.572 \%$ (false rejection rate), which result in the overall factor of the iris verification correctness, equal to $99.5 \%$. For the CASIA database v.1.0 the best result was obtained with the code size of $360 \times 40$ bits and the following results were obtained: $\mathrm{FAR}=3.25 \%, \mathrm{FRR}=3.03 \%$, and the ratio of correct verification of iris codes at the level of $97 \%$ [4].

The best result were received using the IrisBath database by means of the logGabor1D filter. We obtained EER $=0.0031 \%$ for angular span of iris normalization from 120 to $180^{\circ}$. It can be inferred from our experiment that increasing this parameter over $120^{\circ}$ does not improve identification.

Data shown in Table 4 lead to a conclusion that the inner half of the iris area used for normalization contains more distinctive information that the outer half. Another observation is the fact that far inner and far outer parts of iris used for normalization can worsen the identification results because of vicinity of pupil or lids and lashes.

It can be observed that the time of calculations is so short that the proposed iris recognition system can operate in real-time. However, an effective acquisition of the iris image remains a problem.

Open Access This article is distributed under the terms of the Creative Commons Attribution License which permits any use, distribution, and reproduction in any medium, provided the original author(s) and the source are credited.

\section{References}

1. International Organization for Standardization (2005) Biometric data interchange formatspart 6: iris image data, ISO/IEC 19794-6. http://www.iso.org/iso/iso_catalogue/catalogue_ics/ catalogue_detail_ics.htm?csnumber=38750. Accessed 1 Feb 2011

2. Chinese Academy of Sciences' Institute of Automation (2010) "CASIA-IrisV3", http://www. cbsr.ia.ac.cn/IrisDatabase.htm and Biometric Ideal Test website http://biometrics.idealtest.org/. Accessed 15 Feb 2011

3. Boles WW, Boashash B (1998) A human identification technique using images of the iris and wavelet transform. IEEE Trans Signal Process 46(4):1185-1188

4. Dabrowski A et al (2010) D7.3-biometric features analysis component based on video and image information. INDECT Project FP7-218086

5. Daugman JG (1993) High confidence visual recognition of persons by a test of statistical independence. IEEE Trans Pattern Anal Mach Intell 15(11):1148-1161

6. Description of iris acquisition devices: IG-AD100 and IG-H100 (2009) http://www.irisguard.com/ pages.php?menu_id=29\&local_type=0. Accessed 1 Mar 2011

7. Dobeš M, Machala L (2003) Iris database. http://phoenix.inf.upol.cz/iris/. Accessed 15 Feb 2011

8. Field D (1987) Relations between the statistics of natural images and the response properties of cortical cells. J Opt Soc Am A 4(12):2379-2394

9. Iris on the move system (2011) http://sarnoff.com/products/iris-on-the-move, http://www. sarnoff.com/files/IOM_Product_Family_Brochure_20110616_WEB.pdf. Accessed 15 Sep 2011

10. IrisAccess 4000-main brochure (2006) http://www.lgiris.com/download/brochure/ MainBrochure.pdf. Accessed 5 Mar 2011

11. Kaminski T (2007) Implementacja i analiza skutecznosci identyfikacji osob na podstawie teczowki (implementation and efficiency analysis of person identification using iris recognition). M.Sc. Thesis, Supervisor: Tomasz Marciniak, Poznan University of Technology 
12. Kovesi P (2010) Some of my MATLAB functions. http://www.csse.uwa.edu.au/ pk/. Accessed 5 Mar 2010

13. Ma L, Tan T, Wang Y, Zhang D (2003) Personal identification based on iris texture analysis. IEEE Trans Pattern Anal Mach Intell 25(12):1519-1533

14. Ma L, Tan T, Wang Y, Zhang D (2004) Efficient iris recognition by characterizing key local variations. IEEE Trans Image Process 13(6):739-750

15. Masek L (2003) Recognition of human iris patterns for biometric identification. M. thesis, The University of Western Australia

16. Monro DM, Rakshit S, Zhang D (2007) DCT-based iris recognition. IEEE Trans Pattern Anal Mach Intell 29(4):586-596

17. Oki IRISPASS (2006) http://www.oki.com/en/iris/. Accessed 3 Mar 2011

18. Panasonic Iris Reader BM-ET330 (2009) ftp://ftp.panasonic.com/pub/Panasonic/cctv/SpecSheets/ BM-ET330.pdf. Accessed 1 Mar 2011

19. Pereira MB, Paschoarelli Veiga AC (2005) A method for improving the reliability of an iris recognition system. Department of Electrical Engineering, Federal University of Uberlandia (UFU), Brazil

20. Poursaberi A, Araabi BN (2005) A half-eye wavelet based method for iris recognition. In: Proceedings of the ISDA

21. Poursaberi A, Araabi BN (2007) Iris recognition for partially occluded images: methodology and sensitivity analysis. EURASIP J Adv Signal Process 2007(1):20 (article ID 36751)

22. Proença $\mathrm{H}$ et al (2009) The UBIRIS.v2: a database of visible wavelength iris images captured on-the-move and at-adistance. IEEE Trans Pattern Anal Mach Intell. Digital Object Identifier. doi:10.1016/j.imavis.2009.03.003

23. Sanchez-Avila C, Sanchez-Reillo R (2005) Two different approaches for iris recognition using Gabor filters and multiscale zero-crossing representation. Pattern Recogn 38(2):231-240

24. Signal and Image Processing Group (SIPG) (2007) University of Bath Iris Image Database. http://www.bath.ac.uk/elec-eng/research/sipg/irisweb/. Accessed 5 July 2010

25. Vatsa M, Singh R, Noore A (2005) Reducing the false rejection rate of iris recognition using textural and topological features. Int J Signal Process 2(1):66-72

26. Wildes RP (1997) Iris recognition: an emerging biometric technology. Proc IEEE 85(9):13481363

27. Yu L, Zhang D, Wang K, Yang W (2005) Coarse iris classification using box-counting to estimate fractal dimensions. Pattern Recogn 38(11):1791-1798

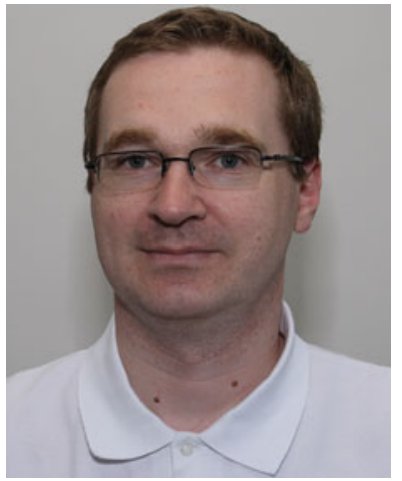

Tomasz Marciniak is an Assistant Professor at the Chair of Control and Systems Engineering of Poznań University of Technology in Poland. He received PhD in Automatics and Robotics in 2003. In 1994, he obtained M.Sc. degree in Electronics and Telecommunications. His research interests include effective implementation of algorithms in biometric and multimedia systems using digital signal processors. As every author of this article, he is engaged in research of FP7 EU project entitled INDECT-Intelligent information system supporting observation, searching and detection for security of citizens in urban environment. 


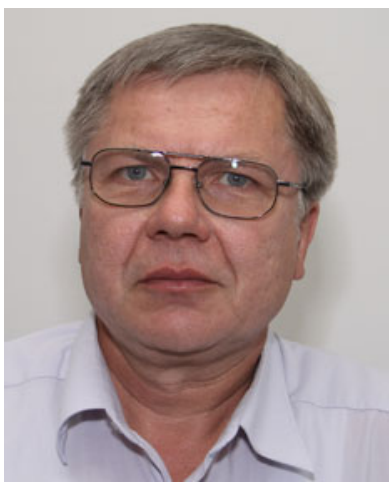

Adam Dąbrowski is a full professor in digital signal processing at the Department of Computing, Poznań University of Technology, Poland. His scientific interests concentrate on: digital signal and image processing (filtering, signal separation, multirate and multidimensional systems, wavelet transformation), multimedia, biometrics, visual systems, and processor architectures. He is author or co-author of 4 books and over 300 scientific papers. He was a Humboldt Foundation fellow at the Ruhr-University Bochum (Germany), visiting professor at the ETH Zurich (Switzerland), Catholic University in Leuven (Belgium), University of Kaiserslautern (Germany), and the Technical University of Berlin (Germany). Currently he is Chairman of the Signal Processing (SP) and Circuits \& Systems (CAS) Chapters of the Poland IEEE Section. In 1995 Professor Adam Dąbrowski won the IEEE Chapter of the Year Award, New York, USA. In 2001 he was also awarded with the diploma for the outstanding position in the IEEE Chapter of the Year Contest.

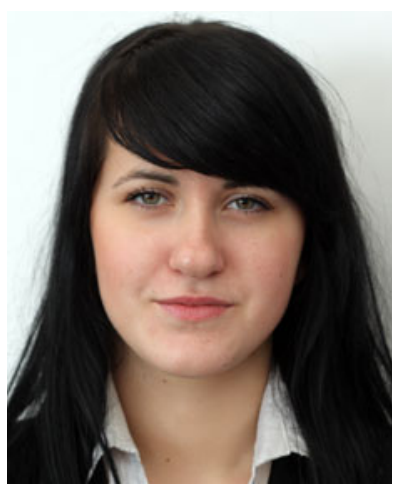

Agata Chmielewska is a PhD student at the Poznań University of Technology (Poland). She received M.Sc. degree in Automatics and Robotics in 2009. She has developed algorithms for the detection of dangerous situations on the basis of video sequences from city monitoring. She also deals with issues of speakers segmentation from the telephone conversation recordings using an acoustic watermark. 


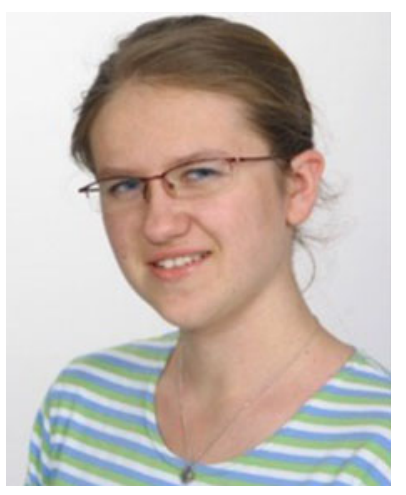

Agnieszka Anna Krzykowska obtained her engineering degree in Automatics and Management at the Poznań University of Technology (Poland) in 2010 and Master degree in Automatics and Robotics majoring in Automatics at the Poznań University of Technology in 2011. Since 2010 she is a PhD student at the Poznań University of Technology under the supervision of Prof. Adam Dąbrowski. Her research interests include audio processing applications, biometric systems and microcontrollers, as well as applications in these areas. 